\title{
Creation of Novel Antihypertensive Agent via Structure-Activity Relationship Study on Phytochemicals towards Vasorelaxant Activity
}

\author{
Yean Chun Loh', Sock Ying Chan', Chuan Wei Oo ${ }^{1 *}$, Mun Fei Yam²
}

'School of Chemical Sciences, Universiti Sains Malaysia, 11800 Minden, Penang, Malaysia

${ }^{2}$ School of Pharmaceutical Sciences, Universiti Sains Malaysia, 11800 Minden, Penang, Malaysia

\section{Key Words \\ plant-derived compounds, phytochemical, structur- al-activity relationship, vascular tone}

\begin{abstract}
Hypertension is defined as a persistently high force of blood flowing through the wall of blood vessels and it has been categorized by the American Heart Association into five stages according to severity. It is a known fact that even the general public terms hypertension as a silent killer in with a big number of public living with it undiagnosed. Even for those that are properly diagnosed and having regular follow-ups, there seem to always be the risk of concomitant diseases such as heart attack and stroke. Moreover, there are reports stated that hypertension is the most frequent co-morbidities in patients infected with COVID-19 that caused by the severe acute respiratory syndrome coronavirus SARSCoV-2 which could lead to high mortality [1].

In facts, antihypertensive drugs have gone through a series of evolution with practitioners switching up different classes of the medication for their patients according to trends of recommendation to assist with achieving a lower risk target blood pressure [2]. There are over hundreds of types of antihypertensive drugs in the current market, but only one-third of the patients that are currently diagnosed have their blood pressure successfully controlled via a monotherapy while the other two-thirds require multiple drug therapy courses to assist them in achieving the same effect. There are
\end{abstract}

Received: April 27, 2020 Reviewed: May 24, 2020 Accepted: June 8, 2020 numerous reports of adverse effects that are due to the long term administration of these drugs, however weighing on its benefits against their risk, there is no better alternative but to allow patients to be exposed to the possibilities of risks. Therefore, there has always been an urgency to search for a better alternative treatment that is either equally or more effective and possess lesser risks than the ones readily available.

Discussion of antihypertensive drug development showed that the vasorelaxant effect of a potential compound should be accessed via the in vitro blood vessels assay before the in vivo testing. Hence, there is a rallying team globally tested for over 250 types of phytochemicals over the past three decades and claimed for their vasorelaxant effects to a great extend, however, only poor numbers of these compounds being adapted into current medical practices [3]. The main concern here would be that even with a potential solution in our hands, there has yet to be action taken to further this cause with all of these researches stuck at the same phase - utilizing similar protocols to analyze different compounds, without any furthering agendas and insights. Furthermore, the current approach seemed to be slightly backdated with its biggest challenge remain at scientists exhausting resources in searching and proving undiscovered compounds that possess the vasorelaxant potential as well as the natural repository for new vasorelaxant compounds discovering could be depleted one day in future.

In present opinions, we suggest the global scientists add value to previous findings conducted by thoroughly investigating the structural-vasorelaxant activity relationship towards the photochemical reported 
and classify the pushing factors (structural characteristics and underlying mechanism of action) that contribute to the high vasorelaxant activity according to their chemical natures [4]. With these pushing factors being revealed, there will be a wider structure-activity profile on vasorelaxant activity available on hands, thus more promising vasorelaxant agents could be synthesized and lead to more options in novel antihypertensive drugs creation that to be applied in current medical practices. Despite blindly searching and testing for the potential vasorelaxant effects of an unknown compound, we strongly believe that the opinions elaborated above could serve better insights for those researching similar topics in the future and potentially lead to at least in the advancement for an alternative antihypertensive study.

\section{Acknowledgements}

Acknowledgements and heartfelt thank you is extended towards the opinions contributed by all co-authors.

\section{Conflict of interest}

The authors declare that there has no conflict of interest.

\section{ORCID}

Yean Chun, Loh http://orcid.org/0000-0002-6274-9098

Sock Ying, Chan. http://orcid.org/0000-0001-5902-5526

Chuan Wei, Oo. http://orcid.org/0000-0002-3666-4007

Mun Fei, Yam. http://orcid.org/0000-0003-4138-711X

\section{Reference}

1. Schiffrin EL, Flack JM, Ito S, Muntner P, Webb RC. Hypertension and COVID-19. Am J Hypertens. 2020;33(5):373-4.

2. Düsing R. Optimizing blood pressure control through the use of fixed combinations. Vascular health and risk management. 2010;6:321.

3. Loh YC, Chan SY, Tew WY, Oo CW, Yam MF. New flavonoid-based compound synthesis strategy for antihypertensive drug development. Life Sci. 2020:117512.

4. Chan SY, Oo CW, Yam MF, Loh YC. Synthesis and Characterization of New Stilbenoid Derivatives as Potential Vasodilators. 7th International Conference for Young Chemists (ICYC 2019), 2020. 